\title{
Lactic acid production from submerged fermentation of broken rice using undefined mixed culture
}

\author{
Luiza Varela Nunes ${ }^{1}$ Fabiane Fernanda de Barros Correa ${ }^{2}$ Pedro de Oliva Neto ${ }^{2}$. \\ Cassia Roberta Malacrida Mayer ${ }^{3} \cdot$ Bruna Escaramboni $^{2} \cdot$ Tania Sila Campioni ${ }^{2}$ Natan Roberto de Barros ${ }^{4}$. \\ Rondinelli Donizetti Herculano ${ }^{4}$ Eutimio Gustavo Fernández Núñez ${ }^{1,5}$
}

Received: 1 November 2016 / Accepted: 6 March 2017 / Published online: 24 March 2017

(C) Springer Science+Business Media Dordrecht 2017

\begin{abstract}
The present work aimed to characterize and optimize the submerged fermentation of broken rice for lactic acid (LA) production using undefined mixed culture from dewatered activated sludge. A microorganism with amylolytic activity, which also produces LA, Lactobacillus amylovorus, was used as a control to assess the extent of mixed culture on LA yield. Three level full factorial designs were performed to optimize and define the influence of fermentation temperature $\left(20-50^{\circ} \mathrm{C}\right)$, gelatinization time (30-60 $\mathrm{min})$ and broken rice concentration in culture medium (40-80 $\mathrm{g} \mathrm{L}^{-1}$ ) on LA production in pure and undefined mixed culture. LA production in mixed culture $\left(9.76 \mathrm{~g} \mathrm{~L}^{-1}\right)$ increased in sixfold respect to pure culture
\end{abstract}

Eutimio Gustavo Fernández Núñez

eutimiocu@yahoo.com

1 Grupo de Engenharia de Bioprocessos, Departamento de Ciências Biológicas, Universidade Estadual Paulista 'Júlio de Mesquita Filho' Campus-Assis, Avenida Dom Antônio, 2100, Assis, SP 19806-900, Brazil

2 Laboratório de Biotecnologia Industrial, Departamento de Biotecnologia, Universidade Estadual Paulista 'Júlio de Mesquita Filho' Campus-Assis, Avenida Dom Antônio, 2100, Assis, SP 19806-900, Brazil

3 Laboratório de Química de Alimentos e Nanobiotecnologia, Departamento de Biotecnologia, Universidade Estadual Paulista "Júlio de Mesquita Filho", Campus-Assis, Avenida Dom Antonio 2100, Bairro Parque Universitário, Assis, SP 19806-900, Brazil

4 Instituo de Química - Araraquara, Universidade Estadual Paulista 'Júlio de Mesquita Filho' Campus-Araraquara, Rua Professor Francisco Degni, 55, Araraquara, SP 14800-900, Brazil

5 Centro de Ciências Naturais e Humanas (CCNH), Universidade Federal do ABC, Avenida dos Estados, 5001, Santo André, SP 09210-580, Brazil in optimal assessed experimental conditions. The optimal conditions for maximizing LA yield in mixed culture bioprocess were $31^{\circ} \mathrm{C}$ temperature, 45 min gelatinization time and $79 \mathrm{~g} \mathrm{~L}^{-1}$ broken rice concentration in culture medium. This study demonstrated the positive effect of undefined mixed culture from dewatered activated sludge to produce LA from culture medium formulated with broken rice. In addition, this work establishes the basis for an efficient and low-cost bioprocess to manufacture LA from this booming agro-industrial by-product.

Keywords Activated sludge $\cdot$ Broken rice $\cdot$ Lactic acid · Mixed culture $\cdot$ Lactobacillus amylovorus $\cdot$ Three-level factorial design

\section{Introduction}

The lactic acid (2-hydroxypropanoic acid), the simplest 2-hydroxycarboxylic acid with a chiral carbon atom, is a valuable chemical compound that has extensive industrial applications in the food, textiles, pharmaceutical, health care and chemical industries (Castillo Martinez et al. 2013; Daful et al. 2016). Recently, the demand of lactic acid (LA) has grown significantly, because of its potential for the chemical synthesis of poly-lactic acid (PLA). Since PLA is a biodegradable polymer, it can be used as an environmentally friendly alternative to petroleum-derived plastics (Upadhyaya et al. 2014). The global demand of LA and PLA are expected to growth annually by 15.5 and $18.8 \%$ to reach 1960.1 and $1205.3 \times 10^{3}$ tons, respectively by 2020 (Abdel-Rahman and Sonomoto 2016).

LA can be produced chemically using petrochemical feedstocks. However, about $90 \%$ of the current commercial LA is obtained via biological fermentation of sugars. 
The main technical advantages of biotechnologies for LA manufacturing lies in their stereospecificity. The production of $\mathrm{D}(-)$-LA or $\mathrm{L}(-)$-LA depends on the strains used. Despite several yeast, fungal strains have been used for LA biological synthesis, the lactic acid bacteria have shown the highest values of yield and productivities for this chemical platform (Subramanian et al. 2015). The cost of culture media based on refined sugars for LA manufacturing using Lactobacillus sps is a key drawback, which has been approached with two major strategies: the use of complex natural starchy raw materials and metabolic engineering (Reddy et al. 2008; Daful et al. 2016). The former alternative involves two branches, two step processes (saccharification-fermentation) and direct conversion using bacteria possessing both amylolytic and LA producing character (Daful et al. 2016). Nevertheless, few amylolytic lactic acid bacteria with the potential to produce efficiently LA are reported until date.

On the other hand, agro-industrial wastes have caused acute environmental problems in both developed and developing countries (Chen et al. 2011). As an example, in Brazil, around $3.25 \times 10^{8}$ t/year of agro-industrial wastes are produced (Maiti et al. 2016). They are mainly composed of proteins, sugars, and lipids along with particular aromatic and aliphatic compounds. In order to overcome this trouble, several solutions have been explored, such as the use of agro-industrial wastes for animal feeding and more recently, the use of processing technologies to fractionate potentially high-value components of them or to generate new high added-value products. Biofuels, bio-pesticides, surfactants, amylases, oligosaccharides have been obtained from agro-industrial wastes by means of biotechnologies (Federici et al. 2009).

Specifically, broken rice, one of the by-products in rice milling, which is usually used for animal feed, is only a third to a half the price of whole rice, its worldwide generation is increasing as the rice production and demand for polished white rice increase (Moin et al. 2016; Setyawati et al. 2016). As a rule, broken rice constitutes $15 \%$ of the rice milled (Chen and Chang 1984). According to FAO's forecast global milled rice production in 2016 will be 496 million ton (FAO 2016). Thus, around 74.4 million ton of broken rice could be generated in the current year. Different technologies have been studied in order to increase the value of this agro-industrial by-product, among the derived products are ethanol, glucoamylase, high-fructose syrup, high-protein flour (Chen and Chang 1984; Anto et al. 2006; Chu-Ky et al. 2016). These technological alternatives are based on chemical composition of broken rice, which comprises mostly starch (74\%) and protein (7\%) (Liu et al. 2016).

The conjunction of environmental and industrial biotechnology, that aim to clean waste and to maximize product yields with high added-value, simultaneously, has led to the potentiation of mixed culture biotechnology, because of this type of cultures is a specific characteristic of biotechnologies originating from the waste treatment field. This new discipline is focused on the effective recovery of specific nutrients or production of specific organic products from wastes. Due to the use of undefined mixed cultures, process development in mixed culture biotechnology should be based on natural/ecological selection by handling bioprocess parameters or modifying the sources of the natural inoculum. The specific advantages of mixed culture biotechnology over classic pure culture in industrial biotechnology include no sterilization requirements, improved adaptive capacity and the ability to use mixed substrates (Kleerebezem and van Loosdrecht 2007). Specifically, the use of undefined mixed culture has been assessed to produce LA from potato peel waste, using sewage sludge as a source of microorganism mixed population. The LA yield was promising $\left(14 \mathrm{~g} \mathrm{~L}^{-1}\right)$ (Liang et al. 2014).

Sewage sludge, difficult waste, is a problem because of the scarcity of treatment strategies for it. Basically, two main methods are used for this purpose, agriculture application and incineration (Dhaouadi and M'Henni 2008). The production of sewage sludge depends on both the quantity of suspended solids and the organic load influent to the treatment systems. The average chemical composition of the sewage sludge comprises $20-25 \%$ dried matter and $60-75 \%, 7$ and $4 \%$ of organic matter, nitrogen, and phosphor, respectively, of the total matter. This type of biosolid contains usually between 11 and $19 \mathrm{~g} \mathrm{~kg}^{-1}$ of the total metals in the dry matter (Galvín et al. 2009). On the other hand, the microbial community in sewage sludge is dominated by Proteobacteria (>50\%). Bacteroidetes, Chloroflexi, and Planctomycetes are also present in a significant number (Wagner et al. 2002; Liang et al. 2015a).

This work aimed to characterize and optimize the submerged fermentation of broken rice for LA production using undefined mixed culture from dewatered activated sludge. The factors included in experimental strategy were gelatinization time of broken rice, broken rice concentration in culture medium and fermentation temperature. A microorganism with amylolytic activity, which also produces LA, Lactobacillus amylovorus, was used as a control to assess the extent of mixed culture on LA yield.

\section{Materials and methods}

\section{Biological agents for biotransformation and inoculum preparation}

The lyophilized strain Lactobacillus amylovorus (ATCC 33620), used for pure cultures, was purchased from the 
Fundação Tropical de Pesquisa e Tecnologia 'André Tosello'- Coleção de Culturas Tropicais, Campinas, SP, Brazil. The revived culture was propagated in De ManRagosa-Sharpe's medium (MRS) (De Man et al. 1972) for inoculum preparation in fermentation experiments and was also kept at $-20{ }^{\circ} \mathrm{C}$ in $10 \%$ glycerol (v/v) to create a local cell bank.

The inoculum used in mixed culture fermentation experiments was dewatered sewage sludge obtained from Wastewater Treatment Station "Limoeiro" (Presidente Prudente, SP, Brazil), which process configuration is classified as extended aeration activated sludge (extended aeration period). The sampling point location was $22^{\circ} 07^{\prime} 04.7^{\prime \prime} \mathrm{S}$ $51^{\circ} 28^{\prime} 09.6^{\prime \prime} \mathrm{W}$ and the sample collection date was on June 30, 2016, at 11:00 a.m. The sample of dewatered sewage sludge was kept chilled in a plastic bag from Wastewater Treatment Station to the Laboratory of Industrial Microbiology (Universidade Estadual Paulista "Júlio de Mesquita Filho", Campus-Assis, SP, Brazil) and the sludge was used immediately in the experimental runs.

\section{Broken rice source and chemical composition}

Broken rice was purchased from a local rice mill (Cerealista Rampazzo Ltda-ME, Cândido Mota, Sao Paulo State, Brazil). The mass percentages (w/w) of moisture (loss on drying: oven drying at $105^{\circ} \mathrm{C}$ until weight constancy), proteins (the Kjeldahl method), lipids (ethylic ether extraction in Soxhlet apparatus) and ashes (incineration at $550{ }^{\circ} \mathrm{C}$ for $4 \mathrm{~h}$ ) of this agro-industrial by-product were determined by classical methods (Zenebon et al. 2008). Each assay was done in triplicate. The carbohydrate content was quantified by the difference between $100 \%$ (w/w) and the sum of the components mentioned above.

\section{Culture media}

The preliminary growth kinetics study was performed for Lactobacillus amylovorus using MRS medium or gelatinized/sterilized broken rice. The broken rice concentration in culture medium and gelatinization time were set according to the specific experimental purpose (Table 1). In all cases, the gelatinization temperature and pressure were $121^{\circ} \mathrm{C}$ and 1 atm gauge pressure, respectively.

\section{Growth kinetics of Lactobacillus amylovorus}

The growth kinetics of Lactobacillus amylovorus in MRS and gelatinized/sterilized broken rice culture media (60 g $\mathrm{L}^{-1}$ and $45 \mathrm{~min}$ gelatinization time) at $35^{\circ} \mathrm{C}$ were monitored over the course of $48 \mathrm{~h}$. Samples for determining cell and reducing sugar concentrations were taken at 0 , $2,4,6,8,10,12,24,36$ and 48 h. Both fermentations
Table 1 Experimental matrix associated to three-level full factorial design for characterizing and optimizing biotransformation of broken rice for LA production

\begin{tabular}{|c|c|c|c|}
\hline Run number & $\begin{array}{l}\text { Fermentation } \\
\text { temperature }\left({ }^{\circ} \mathrm{C}\right)\end{array}$ & $\begin{array}{l}\text { Gelatinization } \\
\text { time (min) }\end{array}$ & $\begin{array}{l}\text { Broken rice } \\
\text { concentration } \\
\left(\mathrm{g} \mathrm{L}^{-1}\right)\end{array}$ \\
\hline 1 & 20 & 30 & 40 \\
\hline 2 & 20 & 30 & 60 \\
\hline 3 & 20 & 30 & 80 \\
\hline 4 & 20 & 45 & 40 \\
\hline 5 & 20 & 45 & 60 \\
\hline 6 & 20 & 45 & 80 \\
\hline 7 & 20 & 60 & 40 \\
\hline 8 & 20 & 60 & 60 \\
\hline 9 & 20 & 60 & 80 \\
\hline 10 & 35 & 30 & 40 \\
\hline 11 & 35 & 30 & 60 \\
\hline 12 & 35 & 30 & 80 \\
\hline 13 & 35 & 45 & 40 \\
\hline 14 & 35 & 45 & 60 \\
\hline 15 & 35 & 45 & 60 \\
\hline 16 & 35 & 45 & 60 \\
\hline 17 & 35 & 45 & 60 \\
\hline 18 & 35 & 45 & 80 \\
\hline 19 & 35 & 60 & 40 \\
\hline 20 & 35 & 60 & 60 \\
\hline 21 & 35 & 60 & 80 \\
\hline 22 & 50 & 30 & 40 \\
\hline 23 & 50 & 30 & 60 \\
\hline 24 & 50 & 30 & 80 \\
\hline 25 & 50 & 45 & 40 \\
\hline 26 & 50 & 45 & 60 \\
\hline 27 & 50 & 45 & 80 \\
\hline 28 & 50 & 60 & 40 \\
\hline 29 & 50 & 60 & 60 \\
\hline 30 & 50 & 60 & 80 \\
\hline
\end{tabular}

were performed in $250 \mathrm{~mL}$ air-locked Erlenmeyer with $200 \mathrm{~mL}$ working volume. The initial cell concentrations were $2.45 \times 10^{6}$ and $0.70 \times 10^{5}$ cell $\mathrm{mL}^{-1}$ (supernatant), for MRS and gelatinized/sterilized broken rice culture medium, respectively. The number of cells inoculated in both systems was $4.90 \times 10^{8}$ cell $\mathrm{mL}^{-1}$. The difference in initial cell concentrations between both systems is caused by cell adherence on gelatinized broken rice.

Cell concentration was measured by hemocytometer method (Neubauer improved counting chamber, Precicolor HBG, Germany) with specified sample dilution using distilled water (Freshney 2010). On the other hand, reducing sugars was determined by an established method (Miller 1959). 
Maximum specific growth rate, $\mu_{\max }$, was calculated by plotting natural logarithm of viable cell concentration versus time during exponential phase and cell productivity was also quantified (Augusto et al. 2010).

\section{Experimental designs for characterization and optimization of biotransformation}

The optimization of biotransformation as well as the influence of fermentation temperature $\left(20-50^{\circ} \mathrm{C}\right)$, gelatinization time (30-60 $\mathrm{min})$ and broken rice concentration (40-80 $\mathrm{g} \mathrm{L}^{-1}$ ) in culture medium on LA production using Lactobacillus amylovorus and mixed culture was assessed according to three-level full factorial design $\left(3^{3}\right)$, adding three repetitions of the central point from experimental domain (Table 1). The fermentation time for Lactobacillus amylovorus and mixed culture were 48 and $336 \mathrm{~h}$, respectively. Biotransformations were performed in 250 $\mathrm{mL}$ air-locked Erlenmeyer with $200 \mathrm{~mL}$ working volume. The inoculum amount was $2.5 \%(\mathrm{v} / \mathrm{v})\left(4.7 \times 10^{7}\right.$ cell $)$ and $2.5 \%(\mathrm{w} / \mathrm{v})$ for Lactobacillus amylovorus and mixed culture fermentations, correspondingly.

\section{Kinetic of lactic acid production using Lactobacillus amylovorus or mixed culture}

The experimental runs with the highest LA production for pure and mixed cultures were kinetically assessed. LA and total acidity were monitored over the course of fermentation. The rate LA to total acidity was calculated for both systems.

\section{Titration of total acidity}

The total acidity in supernatants was quantified by titrating $4 \mathrm{~mL}$ sample volume (diluted in water to $20 \mathrm{~mL}$ ), adding 3 drops $1 \%(\mathrm{w} / \mathrm{v})$ phenolphthalein as indicator, $0.1 \mathrm{M}$ sodium hydroxide $(\mathrm{NaOH})$ was slowly added from a burette into the samples until a pink color appeared. Total titratable acidity was calculated as the following Eq. 1 and expressed as of LA concentration $\left(\mathrm{g} \mathrm{L}^{-1}\right)$ :

Total titratable acidity $\left(\mathrm{gL}^{-1}\right)=\frac{\mathrm{V} \times 90.08 \times \mathrm{M}}{\mathrm{V}}$

where $\mathrm{V}$ is the consumed volume of $0.1 \mathrm{M} \mathrm{NaOH}$ in $\mathrm{mL}$; $\mathrm{M}$ is the molarity of used $\mathrm{NaOH}$ solution $(0.1 \mathrm{M}), \mathrm{v}$ is sample volume $(4 \mathrm{~mL})$ and 90.08 is the LA equivalent $(\mathrm{g})$ for each $\mathrm{mL}$ of $0.1 \mathrm{M} \mathrm{NaOH}$.
Quantification of lactic acid by high-performance liquid chromatography

The quantification of LA in supernatants derived from kinetic studies of best experimental conditions defined through the values of total acidity, both Lactobacillus amylovorus and mixed culture was done by high-performance liquid chromatography (Gey et al. 1990). Briefly, the separation was done in Aminex HPX-87H column $(300 \times 7.8 \mathrm{~mm}$, BioRad, Richmond, CA, EUA) using refractive index detector. The mobile phase was $5 \mathrm{mM}$ sulfuric acid at $0.6 \mathrm{~mL} \mathrm{m^{-1 }}$ flowrate and $50{ }^{\circ} \mathrm{C}$. The sample injection volume was $40 \mu \mathrm{L}$ and the calibration curve was built in $0.25-2.00 \mathrm{~g} \mathrm{~L}^{-1} \mathrm{LA}$ concentrations.

\section{Statistical analysis}

The three-level full factorial design for fermentation characterization (surface response methodology) and optimization (desirability function) was set and analyzed using Design-Expert software (Trial version 9.0.4.1, StatEase, Minneapolis). The statistical decisions in sample comparisons and empiric modeling were taken considering a significance level $(\alpha)$ of 0.05 .

Graphical data treatments from kinetic studies were performed using Excel 2013 (Microsoft Corporation, Redmond, WA). The statistical descriptors: average $\bar{x}$ , standard deviation $(s d)$ and coefficient of variation $\left(\frac{s d}{\bar{x}} \times 100\right)$ when necessary, were calculated in the same software.

The general comparison between LA yield derived from pure and mixed culture was performed according to onetail Student's $t$ test for paired samples $(\alpha=0.05)$. Besides, the rates LA to total acidity for both culture systems were compared according to two-tails Student's $t$ test for independent samples $(\alpha=0.05)$. Both comparative tests were done in Statgraphics Plus 5.0 (Statistical Graphics, Fairfax, VA, USA).

\section{Results}

\section{Chemical composition of broken rice}

The centesimal composition of broken rice was similar to those previously reported for products derived from this cereal (Chen and Chang 1984; Liu et al. 2016). The carbohydrate was the biochemical family with highest mass percentages followed by protein. Lipids are at low levels (Table 2). The dry matter of broken rice utilized in this work was $87.03 \%$ (w/w). 


\section{Growth kinetics of Lactobacillus amylovorus}

The growth and reducing sugars depletion patterns for Lactobacillus amylovorus in MRS medium were typical for pure culture with a non-complex carbon source (glucose). However, the reducing sugar profile shows an increase of reducing sugar over the course of fermentation for Lactobacillus amylovorus in gelatinized broken rice medium. The maximum cell concentrations were $3.11 \times 10^{8}(36 \mathrm{~h})$ and 1.49 $\times 10^{6}$ cell $\mathrm{mL}^{-1}(24 \mathrm{~h})$ in MRS and gelatinized broken rice medium, respectively (Fig. 1); thus, the corresponding cell productivities were $8.57 \times 10^{6}$ and $5.92 \times 10^{4}$ cell $\mathrm{mL}^{-1} \mathrm{~h}^{-1}$.

The $\mu_{\max }$ of Lactobacillus amylovorus in MRS medium was $0.21 \mathrm{~h}^{-1}$, this kinetic parameter was determined in 10-36 hours' interval (exponential growth phase). On the other hand, the $\mu_{\max }$ of the amylolytic microorganism in gelatinized broken rice medium was $0.60 \mathrm{~h}^{-1}(0-4 \mathrm{~h}$ interval).

The lag phase was prolonged (0-12 h) in MRS medium, whereas the cell lysis phase was drastic from $24 \mathrm{~h}$ in the agro-industrial by-product medium (Fig. 1).

\section{Experimental design for submerse fermentation using Lactobacillus amylovorus}

The values of total acidity were in $1.41-3.54 \mathrm{~g}$ of LA L ${ }^{-1}$ for the 30 experimental runs included in three levels experimental design using pure culture (Fig. 2). The quadratic order model was the best-fitted polynomial equation to the collected empiric data. Nevertheless, a reduction of model terms was possible because of the non-significance of several terms in the original quadratic model ( $p$-value $>\alpha=0.05$ ) (Table 3). Both original and modified models were significant and their lack of fit tests were not significant, thereby the modified model in coded factors was used for characterizing the biotransformation under consideration (Eq. 2).

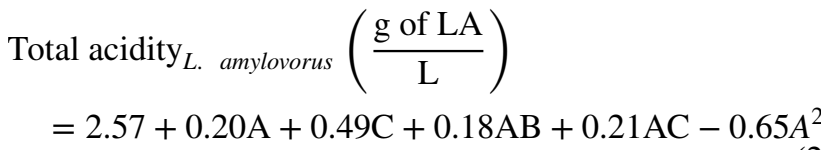

Table 2 Centesimal composition (\%,w/w) of the broken rice used for LA production through pure and mixed cultures

\begin{tabular}{lr}
\hline Chemical component & \multicolumn{1}{l}{$\begin{array}{l}\text { Mass per- } \\
\text { centages }(\%, \\
\text { w/w) }\end{array}$} \\
\hline Moisture & $12.97 \pm 0.17$ \\
Proteins & $7.84 \pm 0.32$ \\
Lipids & $1.21 \pm 0.11$ \\
Ashes & $0.41 \pm 0.05$ \\
Carbohydrates & $77.57 \pm 0.47$ \\
\hline
\end{tabular}

Values are presented in wet basis as the mean \pm standard deviation $(\mathrm{n}=3)$
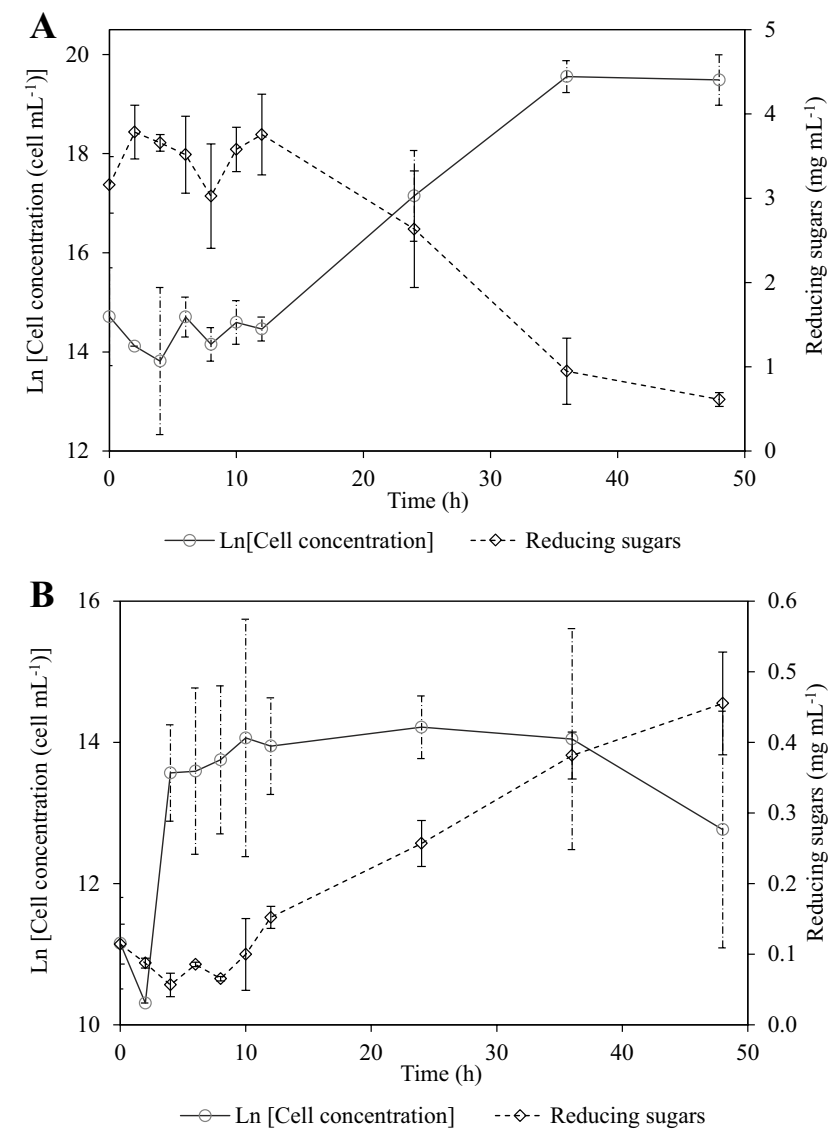

Fig. 1 Growth kinetic and reducing sugar monitoring of Lactobacillus amylovorus fermentation using MRS (a) and gelatinized/sterilized broken rice (b) culture media (the number of repetitions was three and error bars represent the standard deviations)

The gelatinization time (B) did not have a significant impact on the LA production. Conversely, fermentation temperature (A) and concentration of broken rice in culture medium modified markedly the 2-hydroxycarboxylic acid production (Fig. 3a). The fermentation temperature was optimal at $35^{\circ} \mathrm{C}$ and the concentration of broken rice have a linear direct relation with LA production. Minor

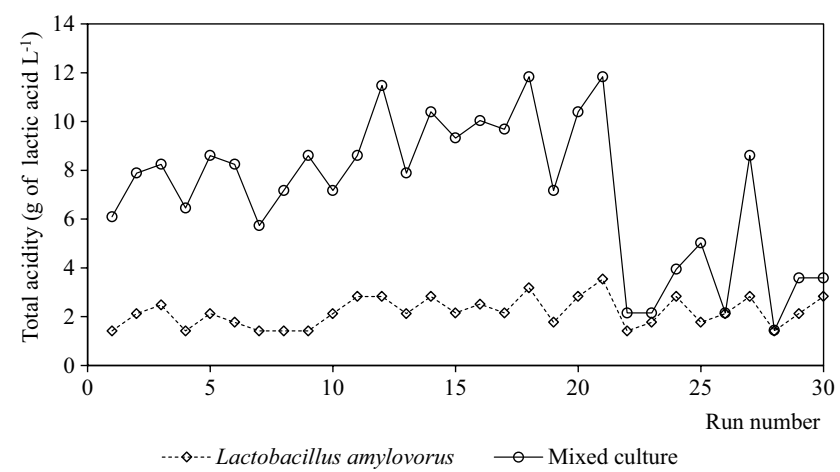

Fig. 2 Primary data for total acidity from $3^{3}$ experimental designs 
Table 3 Analysis of variance (ANOVA) of quadratic models for both three level experimental designs used to describe and optimize statistically the LA production (Total acidity) by Lactobacillus amylovorus and mixed culture

\begin{tabular}{|c|c|c|c|c|c|c|}
\hline \multirow[t]{2}{*}{ Source } & \multicolumn{3}{|c|}{ Lactobacillus amylovorus } & \multicolumn{3}{|l|}{ Mixed culture } \\
\hline & Sum of squares & $\begin{array}{l}\text { Degrees of } \\
\text { freedom }\end{array}$ & $\mathrm{p}$-value & Sum of squares & $\begin{array}{l}\text { Degrees of } \\
\text { freedom }\end{array}$ & p-value \\
\hline Model & 8.990 & 9 & $5.07 \mathrm{E}-06$ & 237.318 & 9 & $9.49 \mathrm{E}-08$ \\
\hline A-temperature & 0.694 & 1 & 0.011551 & 65.831 & 1 & $1.56 \mathrm{E}-06$ \\
\hline B-gelatinization time & 0.062 & 1 & 0.414093 & 0.179 & 1 & 0.730262 \\
\hline C-concentration of broken rice & 4.340 & 1 & $9.52 \mathrm{E}-07$ & 41.259 & 1 & $3.35 \mathrm{E}-05$ \\
\hline $\mathrm{AB}$ & 0.375 & 1 & 0.054449 & 0.096 & 1 & 0.799855 \\
\hline $\mathrm{AC}$ & 0.510 & 1 & 0.027173 & 0.043 & 1 & 0.865718 \\
\hline $\mathrm{BC}$ & 0.000 & 1 & 1 & 0.171 & 1 & 0.73549 \\
\hline $\mathrm{A}^{2}$ & 2.868 & 1 & $1.57 \mathrm{E}-05$ & 109.120 & 1 & $3.46 \mathrm{E}-08$ \\
\hline $\mathrm{B}^{2}$ & 0.000 & 1 & 0.990441 & 7.716 & 1 & 0.032461 \\
\hline$C^{2}$ & 0.000 & 1 & 0.990441 & 0.675 & 1 & 0.504436 \\
\hline Residual & 1.797 & 20 & & 29.215 & 20 & \\
\hline Lack of fit & 1.478 & 17 & 0.667806 & 28.572 & 17 & 0.057515 \\
\hline Pure error & 0.319 & 3 & & 0.643 & 3 & \\
\hline Cor total & 10.787 & 29 & & 266.533 & 29 & \\
\hline
\end{tabular}

synergic effects on LA production were also confirmed for Temperature-Gelatinization time and TemperatureConcentration of broken rice (Eq. 2).

To maximize experimentally LA production $(3.54 \mathrm{~g}$ of LA $\mathrm{L}^{-1}$ ), the fermentation conditions were $35^{\circ} \mathrm{C}$ temperature, 60 min gelatinization time and $80 \mathrm{~g} \mathrm{~L}^{-1}$ broken rice concentration in culture medium (Table 1; Fig. 2). However, the equivalent set of factors determined by desirability function diverged just in fermentation temperature $\left(42^{\circ} \mathrm{C}\right)$ (Table 4$)$.

\section{Experimental design for submerse fermentation using mixed culture from dewatered activated sludge}

The values of total acidity $\left(1.43-11.83 \mathrm{~g}\right.$ of $\left.\mathrm{LA} \mathrm{L} \mathrm{L}^{-1}\right)$ in experimental design runs for mixed culture were higher than those observed for pure cultures (Fig. 2). This was statistically confirmed by one-tail Student's t-test for paired samples $\left(p=2.46805 \times 10^{-13}\right)$. The total acidity values corresponding to the repetitions of the central point in the experimental domain (runs 14-17, Table 1; Fig. 2) were not significantly dispersed (coefficient of variation was $4.7 \%$ ). Like in pure culture, quadratic model was the best-adjusted model to the experimental data (Table 3). The quadratic modified model without significant terms was also significant, thereby, the simplified quadratic model in coded factors was used to describe the LA production in the experimental domain under consideration (Eq. 3).

Total acidity $\left(\frac{\mathrm{g} \text { of LA }}{\mathrm{L}}\right)$

$$
=10.16-1.91 \mathrm{~A}+1.51 \mathrm{C}-3.94 \mathrm{~A}^{2}-1.02 \mathrm{~B}^{2}
$$

Gelatinization time (B) was again the factor with less impact on LA production. The relation type between fermentation temperature-LA production and concentration of broken rice in culture medium-LA production were similar to those confirmed in pure culture system (Fig. 3). Due to the absence of significant interaction terms including two or more factors in the statistical model, no synergic and antagonistic effects among factors were observed in mixed culture fermentation (Eq. 3).

The optimal set of factors to maximize LA production (11.83 $\mathrm{g}_{\left.\text {of } \mathrm{LA} \mathrm{L}^{-1}\right)}$ was observed experimentally at the same combination of factors for pure culture system (Table 1; Fig. 2). Nevertheless, the optimal factor values defined by desirability function were different; specifically, for fermentation temperature and gelatinization time (Table 4).

\section{Kinetic of lactic acid production using Lactobacillus amylovorus and mixed culture from dewatered activated sludge}

As a rule, the LA and total acidity production kinetic profiles showed a similar shape for both systems under study (Fig. 4). The rates LA concentration to total acidity in pure and mixed systems expressed as average \pm standard deviation were $0.62 \pm 0.07$ and $0.76 \pm 0.06$, respectively. Those rates were statistically different $\left(\mathrm{p}=9.5 \times 10^{-4}\right)$. In addition, LA production in mixed culture increased in sixfold respect to pure culture (Fig. 4). 
Design-Expert ${ }^{\circledR}$ Software Factor Coding: Actual

Total acidity (g of Lactic acid/L)

Actual Factors

A: Temperature $=35^{\circ} \mathrm{C}$

B: Gelatinization time $=45 \mathrm{~min}$

$\mathrm{C}$ : Concentration of broken rice $=60 \mathrm{~g} \mathrm{~L}^{-1}$

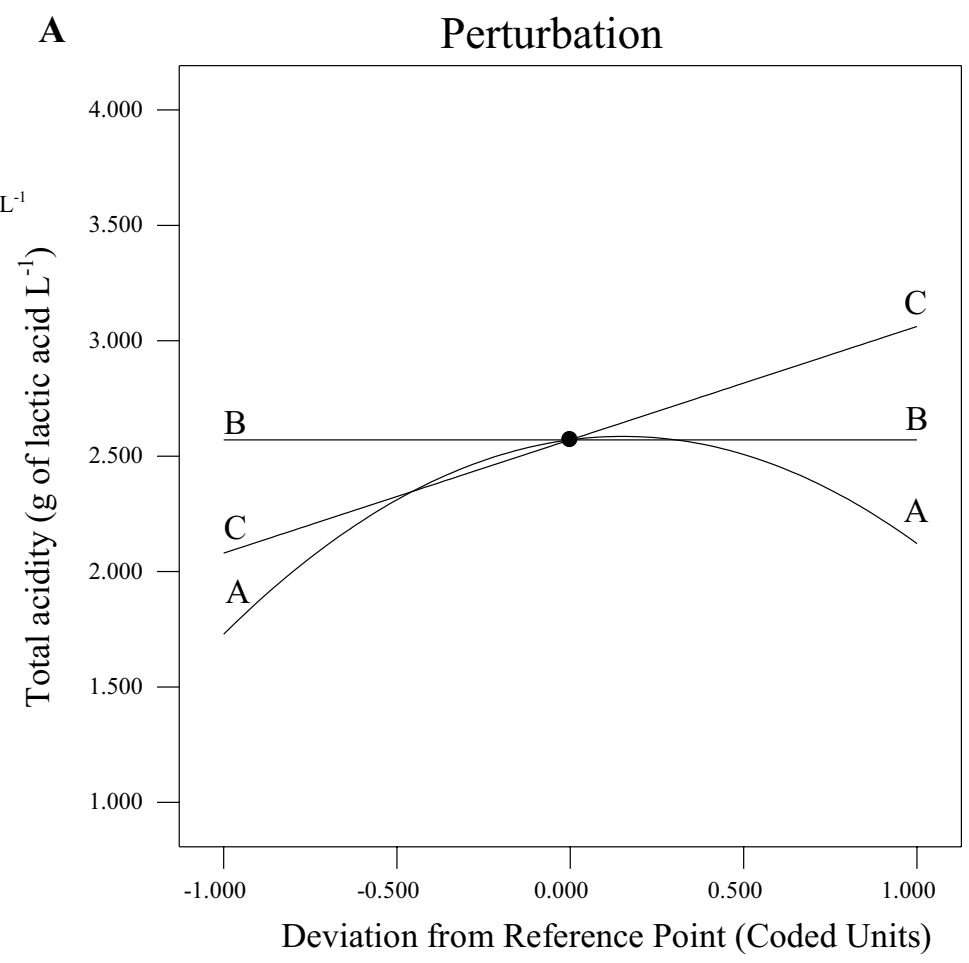

Design-Expert ${ }^{\circledR}$ Software

Factor Coding: Actual

B

Total acidity ( $\mathrm{g}$ of Lactic acid/L)

Actual Factors

A: Temperature $=35^{\circ} \mathrm{C}$

B: Gelatinization time $=45 \mathrm{~min}$

C: Concentration of broken rice $=60 \mathrm{~g} \mathrm{~L}^{-1}$

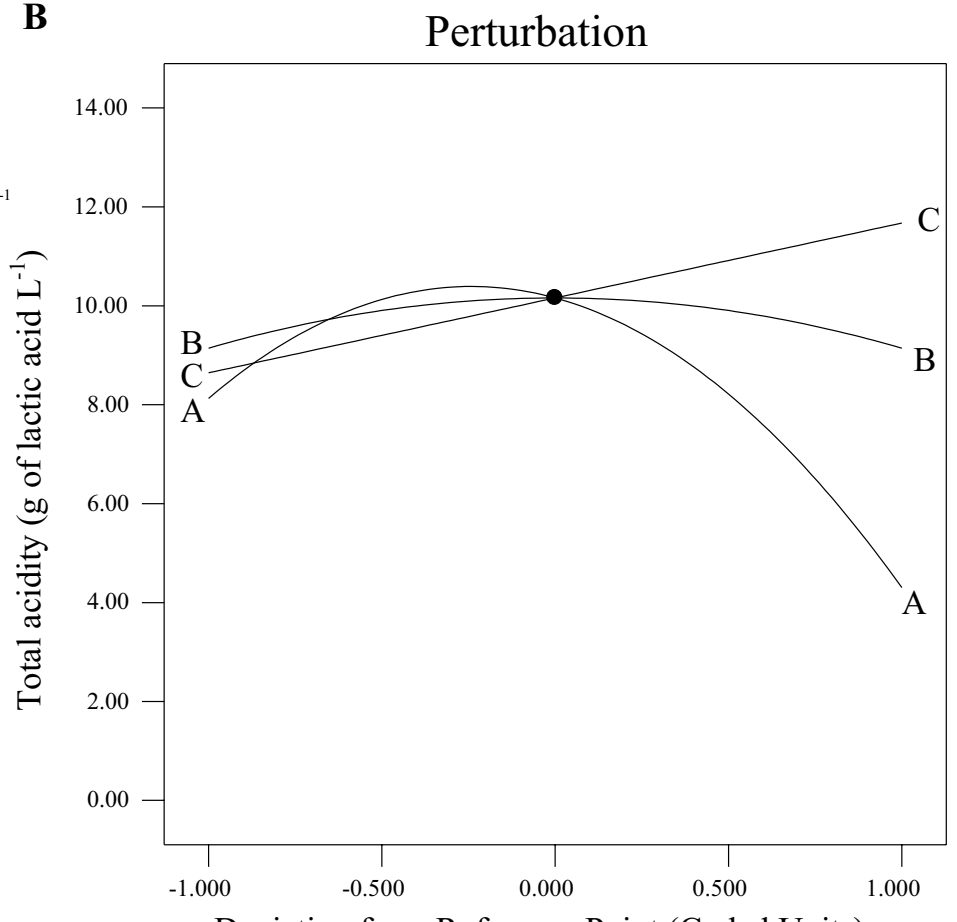

Fig. 3 Perturbation graphs associated to the polynomial models adjusted for describing LA production from gelatinized/sterilized broken rice using Lactobacillus amylovorus (a) and mixed culture (b) 
Table 4 Optimal values for factors under consideration in experimental designs for maximizing LA production

\begin{tabular}{|c|c|c|c|c|c|}
\hline Biological agent & Temperature $\left({ }^{\circ} \mathrm{C}\right)$ & $\begin{array}{l}\text { Gelatinization } \\
\text { time (min) }\end{array}$ & $\begin{array}{l}\text { Concentration of } \\
\text { broken rice }\left(\mathrm{g} \mathrm{L}^{-1}\right)\end{array}$ & $\begin{array}{l}\text { Total acidity } \\
\left(\mathrm{g} \text { of } \mathrm{LA} \mathrm{L}^{-1}\right)\end{array}$ & Desirability \\
\hline L. amylovorus & 41.74 & 60.00 & 80.00 & 3.19 & 0.84 \\
\hline Mixed culture & 31.14 & 44.71 & 79.18 & 11.84 & 1 \\
\hline
\end{tabular}

Desirability function was used as optimization numerical method
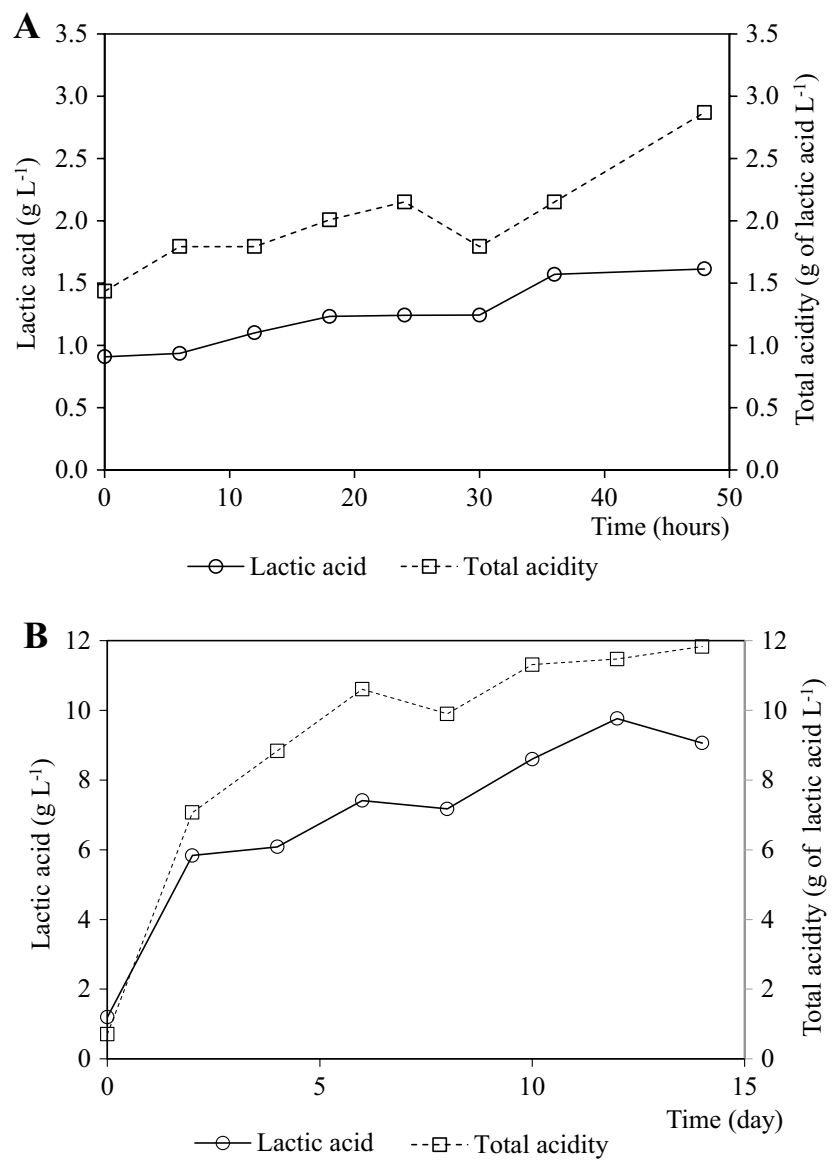

Fig. 4 LA and total acidity kinetics for Lactobacillus amylovorus (a) and mixed culture (b) in optimal assessed conditions (Temperature: $35^{\circ} \mathrm{C}$, gelatinization time: $60 \mathrm{~min}$, broken rice concentration: $80 \mathrm{~g} \mathrm{~L}^{-1}$ )

\section{Discussion}

The two main aspects of the present developing bioprocess, the undefined mixed inoculum from activated sludge and broken rice were carefully chosen taking into account the following arguments. Recently, the use of activated sludge as a source of inoculum for undefined mixed culture has demonstrated robustness and resiliency in LA fermentation from carbohydrate wastes; specifically, from potato peel waste (Liang et al. 2015a). On the other hand, broken rice is a booming agro-industrial by-product with high levels of starch (>70\% w/w) (Liu et al. 2016). Besides, LA rice starch is a better substrate than potato starch for LA fermentation from amylolytic microorganisms (Xiaodong et al. 1997).

Among the amylolytic bacteria for LA production from direct conversion of starchy biomass, Lactobacillus amylovorus stands out (Pompeyo et al. 1993; Reddy et al. 2008). Thus, Lactobacillus amylovorus was chosen as microorganism control to define the real impact of undefined mixed culture on LA production from broken rice. The previous growth kinetic studies were performed in order to confirm indirectly the identity and potential of the Lactobacillus amylovorus strain as well as to define the fermentation time in three-level full factorial design, because of the LA is a growth-associated product (Nakamura 1981).

The Lactobacillus amylovorus strain used in the present work reached a maximum cell concentration equivalent to other culture with similar culture medium $\left(>10^{8}\right.$ cell $\left.\mathrm{mL}^{-1}\right)$ previously reported (Xiaodong et al. 1997). The prolonged lag phase in MRS medium experiment could be explained by the relatively low cell concentration at inoculation. After the lag phase, the time to reach stationary phase was similar to that corresponding to the mentioned previous report $(12 \mathrm{~h})$. In the case of the kinetic experiment carried out with gelatinized/sterilized broken rice culture medium, the increase of reducing sugar over the course of biotransformation confirmed the amylolytic activity of Lactobacillus amylovorus strain utilized in the present work. The reducing sugar concentrations and the time to reach maximum cell concentration herein were in similar order to others already described in equivalent culture medium (Nakamura 1981).

In both assessed full factorial design was used total acidity as the response variable due to the simplicity and low cost of the corresponding analytical technique, also LA is the prevailing acid chemical compound in lactic fermentation (Liang et al. 2014). Acetic acid is also produced (Liang et al. 2015b). The influence of temperature on LA production was analogous for both systems under consideration. The optimal results were obtained around $35^{\circ} \mathrm{C}$, the temperature value reported as optimum both Lactobacillus amylovorus and undefined mixed culture (using potato peel waste and activated sludge as inoculum) fermentations (Nakamura 1981; Liang et al. 2014). The influence of the concentration of broken rice on LA 
production was also similar for pure and mixed culture. A positive linear relationship was confirmed between the concentration of broken rice and LA production. This was already observed in other systems (Liang et al. 2014). This finding could be related to the higher level concentration of low-complex sugars from gelatinization process. However, the influence of gelatinization time in assessed range (30-60 min) was almost negligible on LA production. Thus, this pre-treatment time range of the culture medium did not improve expressively the sugar source availability for the biological agents. On the other hand, the relatively low LA yields in Lactobacillus amylovorus system from broken rice medium could be explained by the absence in the culture medium of additional protein source. This is critical for efficient LA production by Lactobacillus amylovorus when starch is utilized as a carbon source. The LA yields values were equivalent to those reported in a previous work using similar conditions (Mark Hsieh et al. 1999).

The higher LA production in mixed culture respect to pure culture with Lactobacillus amylovorus confirms the synergistic effect among microorganisms in activated sludge to metabolize the rice starch. It is well defined that microbial population (mainly bacteria) of activated sludge is determined by the configuration wastewater treatment systems and the composition of the influent wastewater. Nevertheless, the abundances of those bacteria decrease rapidly. This phenomenon is due to the production of LA ( $\mathrm{pH}$ decrease) and antimicrobial agents by LA bacteria to inhibit or kill the growth of other microorganisms (Liang et al. 2016). The species of the genus Lactobacillus, as a rule, constitute $<1 \%$ in seeds from activated sludge. Nevertheless, the community structures become usually dominated by Lactobacillus sp. over the course of fermentation (>96\%). Among the most abundant Lactobacillus sp. in undefined mixed culture using starch waste are Lactobacillus sp. G24, Lactobacillus gasseri, Lactobacillus mucosae, Lactobacillus amylovorus, Lactobacillus amylolyticus, Lactobacillus panis and Lactobacillus delbrueckii (Liang et al. 2015a). The robustness and resiliency of dewatered activated sludge as inoculum in LA fermentation from broken rice were confirmed in this work by the low coefficient of variation calculated for repetitions of similar bioprocess conditions.

The differences between rates LA concentration and total acidity for pure and mixed cultures demonstrated that more acid species are produced proportionally in Lactobacillus amylovorus than undefined mixed culture. This result reinforces the improving of bioprocess when mixed culture from activated sludge are used to produce LA respect to Lactobacillus amylovorus biotransformation, by reason of product downstream could also make easier.
The optimal values of LA in the present study using broken rice were lower than those reported for potato peel waste in similar conditions (14.7 $\mathrm{g} \mathrm{L}^{-1}$ ) (Liang et al. 2014). This could be explained by a better protein/starch balance in potato peel waste $(0.5)$ than broken rice $(<0.1)$. The positive influence of organic nitrogen source in culture medium on amylolytic bacteria to produce LA has been previously confirmed (Xiaodong et al. 1997; Liang et al. 2014). However, the present work establishes the foundations for LA production at low cost by a bioprocess, which is environmentally friendly. Other elements of this bioprocess, like culture medium supplementation and operation mode, should be studied in details to define a competitive technology lies on broken rice, a growing agricultural by-product.

\section{Conclusions}

This study demonstrated the positive effect of undefined mixed culture from dewatered activated sludge to produce LA from formulated medium containing broken rice. Three operational parameters associated with fermentation process were optimized: $31{ }^{\circ} \mathrm{C}$ temperature, 45 min gelatinization time and $79 \mathrm{~g} \mathrm{~L}^{-1}$ broken rice concentration in culture medium. This work establishes the foundations for a bioprocess development based on mixed culture to manufacture efficiently and low-cost LA from broken rice in order to add value for this booming agro-industrial by-product. In that sense, optimizations of culture medium and operation mode are necessary.

Acknowledgements Authors are grateful to the technical staff of Wastewater Treatment Station "Limoeiro" (Presidente Prudente-SP, Brazil) for donation of dewatered activated sludge. The first author thanks Brazilian National Council for Scientific and Technological Development $(\mathrm{CNPq} / \mathrm{Brazil})$ for Scientific Initiation Scholarship (PIBIC-2015/UNESP/34044). The corresponding author acknowledges his lovely wife, Relma, and daughters, Giovanna and Paola, for the inspiration to write this manuscript.

\section{Compliance with ethical standards}

Conflict of interest The authors declare that they have no conflicts of interest.

\section{References}

Abdel-Rahman MA, Sonomoto K (2016) Opportunities to overcome the current limitations and challenges for efficient microbial production of optically pure lactic acid. J Biotechnol 236:176-192. doi:10.1016/j.jbiotec.2016.08.008

Anto H, Trivedi UB, Patel KC (2006) Glucoamylase production by solid-state fermentation using rice flake manufacturing waste products as substrate. Bioresour Technol 97:1161-1166. doi:10.1016/j.biortech.2005.05.007 
Augusto EFP, Moraes AM, Piccoli RAM et al (2010) Nomenclature and guideline to express the amount of a membrane protein synthesized in animal cells in view of bioprocess optimization and production monitoring. Biologicals 38:105-112. doi:10.1016/j. biologicals.2009.07.005

Castillo Martinez FA, Balciunas EM, Salgado JM et al (2013) Lactic acid properties, applications and production: a review. Trends Food Sci Technol 30:70-83. doi:10.1016/j.tifs.2012.11.007

Chen WP, Chang YC (1984) Production of high-fructose rice syrup and high-protein rice flour from broken rice. J Sci Food Agric 35:1128-1135. doi:10.1002/jsfa.2740351012

Chen L, Yang X, Raza W et al (2011) Solid-state fermentation of agro-industrial wastes to produce bioorganic fertilizer for the biocontrol of Fusarium wilt of cucumber in continuously cropped soil. Bioresour Technol 102:3900-3910. doi:10.1016/j. biortech.2010.11.126

Chu-Ky S, Pham TH, Bui KLT et al (2016) Simultaneous liquefaction, saccharification and fermentation at very high gravity of rice at pilot scale for potable ethanol production and distillers dried grains composition. Food Bioprod Process 98:79-85. doi:10.1016/j.fbp.2015.10.003

Daful AG, Haigh K, Vaskan P, Görgens JF (2016) Environmental impact assessment of lignocellulosic lactic acid production: integrated with existing sugar mills. Food Bioprod Process 99:58 70. doi:10.1016/j.fbp.2016.04.005

De Man J, Rogosa M, Sharpe ME (1972) A medium for the cultivation of lactobacilli. J Appl Bacteriol 23:130-135

Dhaouadi H, M'Henni F (2008) Textile mill effluent decolorization using crude dehydrated sewage sludge. Chem Eng J 138:111119. doi:10.1016/j.cej.2007.05.052

FAO (2016) http://www.fao.org/fileadmin/templates/est/COMM_ MARKETS_MONITORING/Rice/Images/RMM/RMM_JUL16. pdf. July 2016, Accessed 29 Sept 2016

Federici F, Fava F, Kalogerakis N, Mantzavinos D (2009) Valorisation of agro-industrial by-products, effluents and waste: concept, opportunities and the case of olive mill waste waters. J Chem Technol Biotechnol 84:895-900. doi:10.1002/jctb.2165

Freshney R (2010) Culture of animal cells: a manual of basic technique and specialized applications. 6th edn, Wiley, Hoboken

Galvín RM, López JMC, Mellado JMR (2009) Chemical characterization of biosolids from three Spanish WWTPs: transfer of organics and metallic pollution from urban wastewater to biosolids. Clean - Soil, Air, Water 37:52-59. doi:10.1002/clen.200800154

Gey M, Klosser P, Becker U (1990) Characterization of biotechnological processes and products using high-performance liquid chromatography (HPLC): VI. Determination of lactic acid and shortchain carboxylic acids $\mathrm{C}_{1}-\mathrm{C}_{5}$. Acta Biotechnol 10:459-468

Kleerebezem R, van Loosdrecht MC (2007) Mixed culture biotechnology for bioenergy production. Curr Opin Biotechnol 18:207212. doi:10.1016/j.copbio.2007.05.001

Liang S, McDonald AG, Coats ER (2014) Lactic acid production with undefined mixed culture fermentation of potato peel waste. Waste Manag 34:2022-2027. doi:10.1016/j.wasman.2014.07.009

Liang S, Gliniewicz K, Mendes-Soares H et al (2015a) Comparative analysis of microbial community of novel lactic acid fermentation inoculated with different undefined mixed cultures. Bioresour Technol 179:268-274. doi:10.1016/j.biortech.2014.12.032
Liang S, McDonald AG, Coats ER (2015b) Lactic acid production from potato peel waste by anaerobic sequencing batch fermentation using undefined mixed culture. Waste Manag 45:51-56. doi:10.1016/j.wasman.2015.02.004

Liang S, Gliniewicz K, Gerritsen AT, McDonald AG (2016) Analysis of microbial community variation during the mixed culture fermentation of agricultural peel wastes to produce lactic acid. Bioresour Technol 208: 7-12. doi:10.1016/j. biortech.2016.02.054

Liu H, Wan H, Xu S et al (2016) Influence of extrusion of corn and broken rice on energy content and growth performance of weaning pigs. Anim Sci J. doi:10.1111/asj.12578

Maiti S, Sarma SJ, Brar SK et al (2016) Agro-industrial wastes as feedstock for sustainable bio-production of butanol by Clostridium beijerinckii. Food Bioprod Process 98:217-226. doi:10.1016/j.fbp.2016.01.002

Mark Hsieh C, Yang FC, Iannotti EL (1999) The effect of soy protein hydrolyzates on fermentation by Lactobacillus amylovorus. Process Biochem 34:173-179. doi:10.1016/S0032-9592(98)00081-8

Miller GL (1959) Use of dinitrosalicylic acid reagent for determination of reducing sugar. Anal Chem 31:426-428

Moin A, Ali TM, Hasnain A (2016) Effect of succinylation on functional and morphological properties of starches from broken kernels of Pakistani Basmati and Irri rice cultivars. Food Chem 191:52-58. doi:10.1016/j.foodchem.2015.03.119

Nakamura LK (1981) Lactobacillus amylovorus, a new starch-hydrolyzing species from cattle waste-corn fermentations. Int J Syst Bacteriol 31:56-63

Pompeyo C, Gómez M, Gasparian S, Morlon-Guyot J (1993) Comparison of amylolytic properties of Lactobacillus amylovorus and of Lactobacillus amylophilus. Appl Microbiol Biotechnol 40:266-269. doi:10.1007/BF00170378

Reddy G, Altaf M, Naveena BJ et al (2008) Amylolytic bacterial lactic acid fermentation-a review. Biotechnol Adv 26:22-34. doi:10.1016/j.biotechadv.2007.07.004

Setyawati YD, Ahsan SF, Ong LK et al (2016) Production of glutinous rice flour from broken rice via ultrasonic assisted extraction of amylose. Food Chem 203:158-164. doi:10.1016/j. foodchem.2016.02.068

Subramanian MR, Talluri S, Christopher LP (2015) Production of lactic acid using a new homofermentative Enterococcus faecalis isolate. Microb Biotechnol 8:221-229. doi:10.1111/1751-7915.12133

Upadhyaya BP, DeVeaux LC, Christopher LP (2014) Metabolic engineering as a tool for enhanced lactic acid production. Trends Biotechnol 32:637-644. doi:10.1016/j.tibtech.2014.10.005

Wagner M, Loy A, Nogueira R et al (2002) Microbial community composition and function in wastewater treatment plants. Antonie Van Leeuwenhoek 81:665-680. doi:10.102 3/a:1020586312170

Xiaodong W, Xuan G, Rakshit SK (1997) Direct fermentative production of lactic acid on cassava and other starch substrates. Biotechnol Lett 19:841-843

Zenebon O, Pascuet NS, Tiglea P (2008) Métodos físico-químicos para análise de alimentos. Instituto Adolfo Lutz, São Paulo (online version) 